\title{
Cardiac Valvular Regurgitation Indicator
}

National Cancer Institute

\section{Source}

National Cancer Institute. Cardiac Valvular Regurgitation Indicator. NCI Thesaurus. Code C139055.

An indication as to whether a specific cardiac valve is regurgitant. 